\section{GROUNDBREAKING IMPLANTOLOGY E-LEARNING}

The International Team for Implantology (ITI), the leading academic organisation dedicated to promoting education and research in implant dentistry, has launched its most significant educational offering: the ITI Online Academy.

The Academy combines high quality, evidencebased content with flexibility and ease of use to deliver a motivating and rewarding learning experience. A comprehensive peer-reviewed curriculum of modules addresses users at all levels, featuring lectures and case studies that combine to provide focused learning pathways.

Open to everyone with an interest in implant dentistry, online registration is free; just go to http://academy.iti.org.

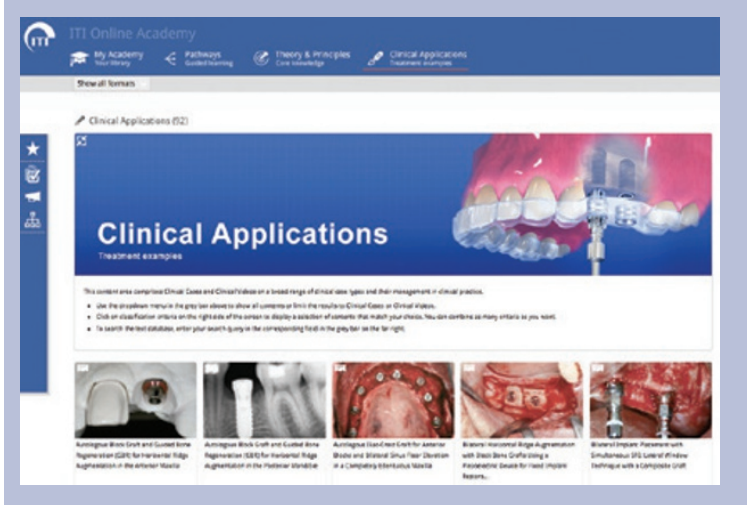

\section{A TRULY INJECTABLE COMPOSITE}

GC UK has produced a fabulous full colour brochure which provides you with the latest tips and tricks for using the world's first truly injectable composite: Gaenial Universal Flo.

GC's ongoing commitment to research in highly wear resistant coatings has driven the innovation behind the new level of wear resistance that can be achieved with Gaenial Universal Flo. The high filler loading (69\% wt) and homogenous dispersion of filler required for a high strength universal composite was achieved through the introduction of new manufacturing processes. A new de-foaming process delivers porosity-free composite that further improves strength and enhances the final polish.

Gaenial injects like a flowable material, yet stacks like a traditional composite meaning that the composite placement is exact and controlled making the build-up of anatomical form easy to achieve. Controlled fluidity ensures placement is quick and stress-free. The combination of smooth flow and thixotropic properties means Gaenial Universal Flo wets surfaces easily, but is not runny and stays neatly in place. Gaenial Universal Flo offers a fast placement technique for all cavity designs with quick finishing and a beautiful aesthetic end result.

Gaenial Universal Flo gives you and your patients the highest level of aesthetics. Your patients will appreciate the self-polishing characteristics, where the high gloss finish is maintained with daily toothbrushing.

To receive your free copy of a Touch of Genius, or for further information, contact GC UK on 01908218999.

\section{NEW VERIFIABLE CPD COURSES AVAILABLE}

Colgate Professional invites you to explore their library of verifiable continuing professional development (CPD) materials and take advantage of other valuable educational resources. Visit www.colgateprofessional. co.uk and click on the 'Professional Education' button to gain verifiable CPD on a wide range of topics including:

Care for Caries in Older Patients: View an educational symposium, providing evidence based and practical advice on effective prevention and management of caries within an ageing patient base.

A Professional's insight into the Latest Electric Toothbrushing Technology: This presentation covers current concepts in toothbrushing, challenges in achieving optimal oral hygiene, risk factors associated with ineffective toothbrushing, how the latest innovation from Colgate can assist patients with more effective toothbrushing, and how the Colgate ProClinical electric toothbrush range can improve the oral health of patients.

DHS in the Dental Surgery: Join Professor David Bartlett to learn more about how to manage DHS with this interesting and informative overview including personal experiences of dentine hypersensitivity and how his own perceptions around risk factors have evolved. This broadcast also looks at epidemiology and aetiology before moving to reviewing measurement methods and underlying conditions as contributing factors.

In addition to $\mathrm{CPD}$, the Colgate Professional website provides a wealth of information and resources to support your clinical practice including educational materials for patients, detailed product information, free samples and the latest oral health news.

Get started today by visiting www.colgateprofessional.co.uk.

\section{THE CRUCIAL EARLY DIAGNOSIS OF TOOTH WEAR}

On 9 June 2014, GSK Consumer Healthcare, the manufacturers of Pronamel, held a meeting of dental professionals and academics. The meeting discussed ground-breaking data from an ESCARCEL study which was sponsored by GSK. This study revealed that $29.4 \%$ of young European adults aged 18-35 years already have moderate tooth wear. ${ }^{1}$

To help dental professionals in the crucial early diagnosis of tooth wear, the meeting suggested the use of the Basic Erosive Wear Examination (BEWE), which facilitates consistent measurement and recording of tooth wear. The duration over which acid is consumed and method of consumption must be considered in risk management. The BEWE is featured in the 2014 Delivering better oral health toolkit as a method for the prevention of acid wear. ${ }^{2}$

Visit www.gsk-dentalprofessionals.co.uk.

1. Bartlett D W, Lussi A, West N X, Bouchard P, Sanz M, Bourgeois D. Prevalence of tooth wear on buccal and lingual surfaces and possible risk factors in young European adults. J Dent 2013; 41: 1007-1013.

2. Department of Health. Delivering better oral health. An evidencebased toolkit for prevention, 3rd ed. 2014. 